\title{
GESTORES DO ESPORTE: REFLEXÕES SOBRE SUA FORMAÇÃO E DESENVOLVIMENTO PROFISSIONAL
}

\author{
Ricardo Teixeira Quinaud \\ Universidade Federal de Santa Catarina, Florianópolis, Santa Catarina, Brasil. \\ Leandro Carlos Mazzei \\ Universidade de Campinas, Campinas, São Paulo, Brasil. \\ Fabrício João Milan \\ Universidade Federal de Santa Catarina, Florianópolis, Santa Catarina, Brasil. \\ Michel Milistetd \\ Universidade Federal de Santa Catarina, Florianópolis, Santa Catarina, Brasil. \\ Juarez Vieira do Nascimento \\ Universidade Federal de Santa Catarina, Florianópolis, Santa Catarina, Brasil.
}

\begin{abstract}
Resumo
O objetivo do presente ensaio foi refletir sobre a formação e o desenvolvimento profissional de gestores do esporte, tendo como base a Teoria da Aprendizagem de Knud Illeris. Inicialmente, houve a preocupação de abordar o gestor esportivo e suas competências. Na sequência, ressaltou-se que as dimensões de conteúdo, incentivo e interação são imprescindíveis para que ocorra o desenvolvimento profissional dos gestores. Enquanto no ambiente educacional, a formação do gestor esportivo no Brasil concentra-se nos cursos de graduação em Educação Física e Esporte, o ambiente de trabalho é onde irão desenvolver aprendizagens específicas para a sua atuação. Esperamos que este ensaio estimule a reflexão dos leitores sobre as aprendizagens em que o gestor esportivo está inserido, bem como possa suscitar novos questionamentos e avanços na área da gestão do esporte.
\end{abstract}

Palavras-chave: Administração. Aprendizagem. Educação.

\section{SPORTS MANAGERS: REFLECTIONS ABOUT THEIR EDUCATION AND PROFESSIONAL DEVELOPMENT}

\begin{abstract}
The aim of this essay was to reflect on the formation and professional development of sport managers, based on the Knud Illeris Learning Theory. Initially there was the concern to approach the sports manager and his competences. It was pointed out that content, incentive and interaction dimensions are essential for the development of manager's professional learning. While in the educational space, the development of sports managers in Brazil focuses on undergraduate majors of Physical Education and Sport, the work space is where they will develop specific learning for their action. We hope that this essay will stimulate the readers' reflection on the learning that the sports manager is inserted, as well as may raise new questions and advances in the area of sports management.
\end{abstract}

Keyword: Administration. Learning. Education. 


\title{
GESTORES DEL DEPORTE: REFLEXIONES SOBRE SU FORMACIÓN Y DESARROLLO PROFESIONAL
}

\begin{abstract}
Resumen
El objetivo del presente ensayo fue reflexionar sobre la formación y desarrollo profesional de gestores del deporte, tomando como base la Teoría del Aprendizaje de Knud Illeris. La preocupación inicial fue tratar el tema del gestor deportivo y sus habilidades. Enseguida se resaltó que las dimensiones de contenido, incentivo e interacción son imprescindibles para que ocurra el desarrollo profesional de los gestores. Mientras que, en el ambiente educativo, la formación del gestor deportivo en Brasil se concentra en los cursos de las carreras de Educación Física y Deporte, el ambiente de trabajo es donde se van a desarrollar aprendizajes específicos para su actuación. Esperamos que este ensayo estimule la reflexión de los lectores sobre los aprendizajes en los que el gestor deportivo está involucrado, así como también, suscitar nuevos cuestionamientos y avances en el área de la gestión del deporte.
\end{abstract}

Palabras clave: Administración. Aprendizaje. Educación.

\section{Introdução}

A crescente atenção voltada à gestão do esporte, em especial pela falta dela na chamada "década dos megaeventos esportivos no Brasil" (MAZZEI; ROCCO JÚNIOR, 2017), fizeram com que temas relacionados com a formação e o perfil do gestor esportivo voltassem a receber determinada atenção por parte de diversos setores, incluindo a mídia, a opinião pública e também a academia. Alguns desses temas envolvem competências (JOAQUIM; BATISTA; CARVALHO, 2011; AMARAL; BASTOS, 2015), como as habilidades gerenciais (MOCSÁNYI; BASTOS, 2005; SORDI; THEOBALD, 2017), que os gestores necessitam dominar. Para tanto, a procura por uma formação profissional, junto a conteúdos coerentes com a gestão contemporânea do esporte, vem se tornando intensa em publicações nacionais recentes (MAZZEI; AMAYA; BASTOS, 2013; CÁRDENAS; FEUERSCHÜTTE, 2015; JÚNIOR; PADEIRO, 2018).

Uma formação de qualidade do gestor esportivo tornou-se necessária, principalmente no momento em que o esporte e também a sua gestão se tornam cada vez mais globais e profissionais (SKINNER; GILBERT, 2007). A contratação de pessoas qualificadas se tornou uma obrigação para as diversas organizações que atuam no âmbito esportivo, sendo necessário que esse profissional seja um conhecedor considerável de conceitos essenciais para a sua atuação, além do ambiente em que atua (SCHWAB et al., 2015). Na compreensão moderna do esporte, sempre houve mais do que discussões sobre aspectos físicos, biológicos e financeiros, e que hoje são ainda mais visíveis na sociedade contemporânea (BENTO; CONSTANTINO, 2007). $\mathrm{O}$ fenômeno esporte envolve todo um sistema vigente de valores culturais. Esses valores também estão presentes na sua prática, na inspiração de muitos indivíduos que procuram o esporte e que não devem ser desvalorizados por suas organizações (PIRES, 2005). Por sua vez, como em qualquer outro campo de atuação, tais conhecimentos perpassam pela aquisição de aprendizagens (CONKLIN; KYLE; ROBERTSON, 2012).

A aprendizagem é caracterizada como um processo complexo, sem uma definição conceitual única, cuja variedade de perspectivas transita por diversos ambientes, sendo essas perspectivas essencialmente abertas. Tal conceito surge em decorrência de sua aplicação como teoria, especialmente ao não se limitar a cenários específicos, o que institui a importância que esses prováveis cenários têm para a aprendizagem, não sendo possível desconsiderar os que apresentam pontos de partida (história de vida, escola, trabalho, entre outros) e os que não 
apresentam (ILLERIS, 2007). Essa visão heterogênea em face da aprendizagem também permite distintas abordagens teóricas sobre ela, sendo estas constituídas como importantes elementos da contemporaneidade e que debruçam um novo olhar sobre as condições resultantes do aprender.

Diante do exposto, o presente ensaio tem como objetivo refletir sobre a formação e o desenvolvimento profissional de gestores do esporte, tendo como base a Teoria da Aprendizagem de Knud Illeris. Knud Illeris, nascido em 1939, é professor dinamarquês de aprendizagem ao longo da vida. Sua teoria da aprendizagem tem abordagem, na qual ele mesmo se identifica, como psicológica no desenvolvimento da aprendizagem e da identidade das pessoas. É a primeira vez que sua teoria, original do campo de estudos da educação, é utilizada na compreensão da aprendizagem de gestores esportivos, o que nos faz ter que refletir mais profundamente em razão de ser um novo campo de compreensão ainda não estudado.

Esperamos que o leitor, ao final deste ensaio teórico, possa compreender sobre as possibilidades de aprendizagem profissional do gestor esportivo, cujas indagações possam estimular a realização de novas pesquisas, bem como ampliar as discussões e as proposições para a melhoria da qualidade da gestão do esporte no Brasil.

\section{O gestor esportivo e suas competências}

A função do gestor esportivo geralmente envolve a responsabilidade de buscar um esporte melhor organizado em meio à sociedade atual (SMART, 2007). Ao considerarmos a exposição do Brasil devido à realização de megaeventos internacionais no país na última década, foi identificada a necessidade de melhor profissionalização ou, em outras palavras, aumentar a qualidade dos conhecimentos (teórico-práticos) de nossos gestores na condução do esporte brasileiro, no sentido de ser melhor organizado, responsável e sustentável, assim como formar melhores e mais capacitados gestores esportivos no país (MAZZEI; AMAYA; BASTOS, 2013).

No campo acadêmico, a tendência das publicações seguiu a linha de descrição do perfil do gestor esportivo. Estudos foram publicados sobre o perfil de gestores de clubes e federações, de diferentes modalidades, gestores públicos e do setor de fitness, dentre outros. No geral, esses estudos contemplaram tanto o âmbito público como privado do universo de organizações esportivas existentes (JOAQUIM; BATISTA; CARVALHO, 2011; AMARAL; BASTOS, 2015; FELIX; FERNANDES; OLIVEIRA, 2015; FREITAS et al., 2015; FREITAS; GIRGINOV; TEOLDO, 2016). Além disso, também podemos dizer que, na busca pelo entendimento de quem é esse profissional, foi identificado que os processos de aprendizagem pelos quais esses profissionais passaram são polissêmicos, em que a sua efetivação no cargo de gestor se deu por inúmeros motivos (indicação, progressão de carreira, paixão pelo esporte, entre outros), e não estavam necessariamente ligados às competências necessárias à sua função (JOAQUIM; BATISTA; CARVALHO, 2011; AMARAL; BASTOS, 2015; FELIX; FERNANDES; OLIVEIRA, 2015; FREITAS et al., 2015; FREITAS; GIRGINOV; TEOLDO, 2016)

A partir da leitura e da interpretação de estudos, a Figura 1 contempla um resumo das informações que descrevem o perfil do gestor esportivo no Brasil. 
Figura 1: Perfil do gestor esportivo

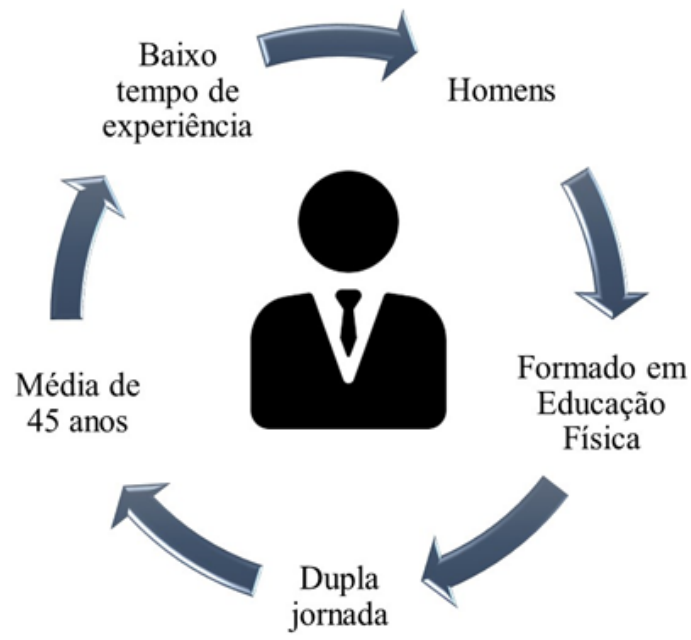

Fontes: Azevedo, Barros e Suaiden (2004); Bastos et al. (2006); Karnas (2010); Barros Filho et al. (2013); Gomes, Sarmento e Mulatinho (2014); Amaral e Bastos (2015).

Especificamente sobre as competências desses gestores esportivos, esta foi abordada sobre a ótica de um "produto" de sua progressão no desempenho dessa atividade. Na tentativa de auxiliar na compreensão das diversas competências dos gestores esportivos, a Figura 2 apresenta a síntese dos resultados de estudos originais e de revisões, com ênfase nos estudos nacionais, mas também considerando alguns conceitos provenientes de estudos internacionais.

Figura 2: Sistematização das competências do gestor esportivo de acordo com as dimensões de competência técnica, contextual e comportamental

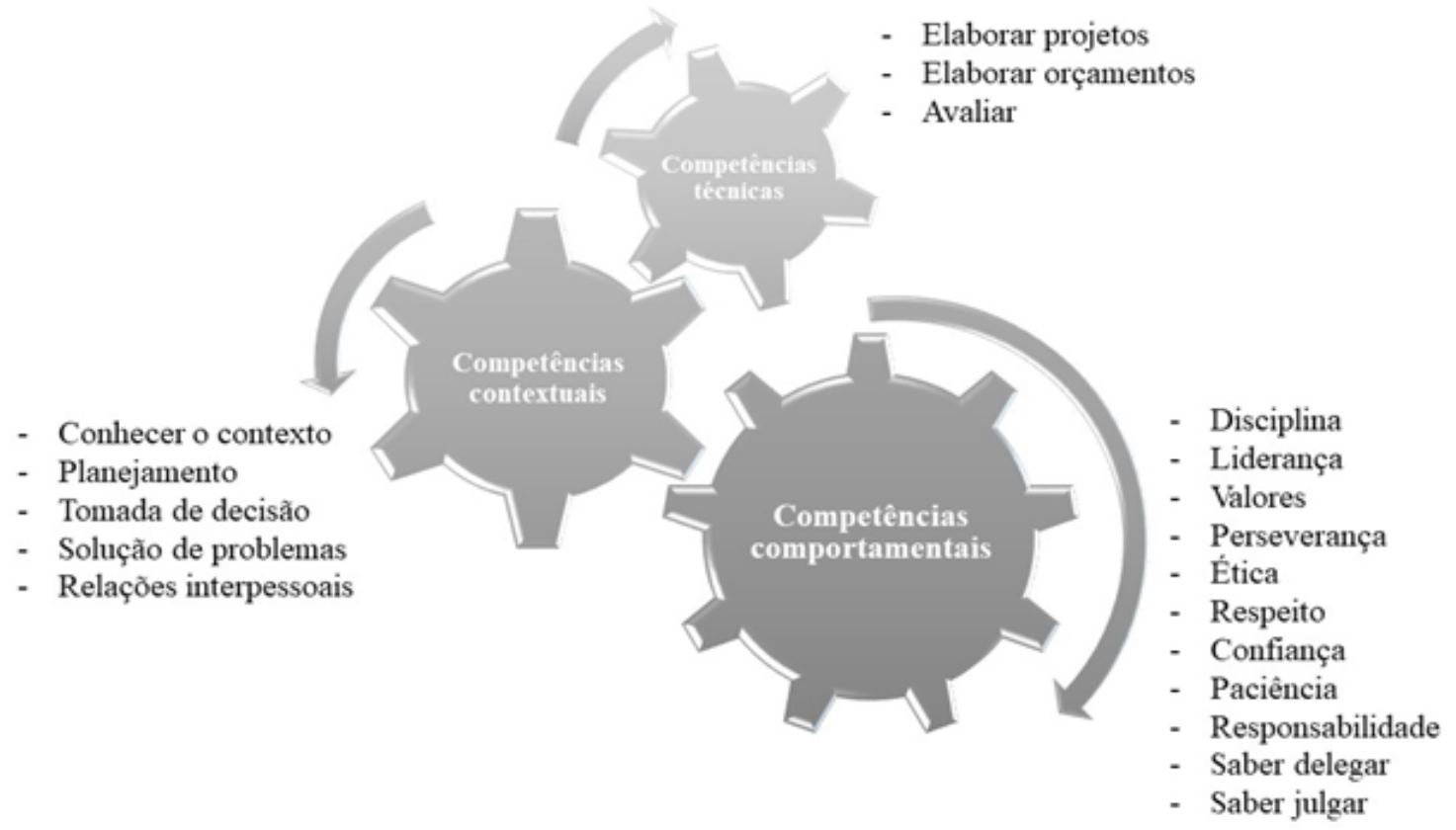

Fontes: Milne (2007); Tavares (2010); Pacesa, Zakarevicius e Zukauskas (2011); Joaquim, Batista e Carvalho (2011); Mello e Silva (2013); Gomes, Sarmento e Mulatinho (2014); Amaral e Bastos (2015); Felix, Fernandes e Oliveira (2015); Marjoribanks e Farquharson (2016); Vilhelmsdottir, Kristjansdottir e Ingason (2016); Miranda et al. (2017). 
As competências apresentadas e coletadas a partir das publicações sobre o tema foram subdivididas em três dimensões: técnicas, contextuais e comportamentais. As competências técnicas são consideradas elementos e aprendizagens procedimentais que disponibilizam "ferramentas" ao gestor para elaboração e avaliação de, por exemplo, projetos esportivos. Por sua vez, as competências contextuais envolvem as inter-relações entre o gestor e a organização. A terceira dimensão é compreendida como competências inerentes ao sujeito e ao seu comportamento pessoal. As três competências e seus respectivos elementos não são isolados, logo, apesar de por vezes alguma se sobressair sobre as outras, elas se interagem e são particulares ao tamanho da função e/ou da organização (CAUPIN et al., 2006).

Infelizmente, a gestão do esporte ainda não ocorre de forma profissional no Brasil, com pessoas que direcionaram diferentes atuações e formações não específicas para essa finalidade. A função de gestor esportivo vem sendo exercida por ex-professores, treinadores e uma grande variedade de outros profissionais que, por falta de recursos humanos especializados, acabam por assumir esse papel de gestor. Não desprezamos a atuação desses outros profissionais na gestão do esporte, mas é fato que, na maioria dos casos, não houve uma formação ou uma capacitação para a aquisição de conhecimentos específicos da ciência da administração; ou mesmo por administradores que não têm conhecimentos suficientes na área da Educação Física e do Esporte (MOCSÁNYI; BASTOS, 2005). Para desenvolver uma gestão do esporte adequada, há necessidade da junção do conhecimento e das competências necessárias, realizando a articulação das aprendizagens já adquiridas com as novas, bem como conduzindo interpretações corretas e tomadas de decisões a serem colocadas em prática (SILVA, 2004). De fato, recomendamos que o gestor esportivo seja um profundo conhecedor do ambiente no qual atua, o que permitirá à gestão do esporte obter sentido e ganhar credibilidade para assegurar maiores possibilidades de intervenção profissional (MAZZEI et al., 2012).

A partir do contexto descrito, alguns questionamentos sobre o gestor esportivo vão na seguinte direção: onde e/ou como deveriam ocorrer as aprendizagens das competências que norteiam a atuação dos gestores esportivos? No decorrer deste ensaio, abordaremos alguns conceitos que podem subsidiar as respostas para essas e demais perguntas sobre a formação e a aprendizagem que envolvem os gestores esportivos.

\section{Aprendizagem profissional de gestores esportivos}

A compreensão geral sobre aprendizagem é de que esta se manifesta como uma questão complexa, pois é preciso considerar vários ambientes em que ela ocorre (ILLERIS, 2007). Para Jarvis (2006; 2007; 2008), o processo de aprendizagem ao longo da vida indica que o ser humano aprende e se transforma desde seu nascimento até sua morte. Portanto, a interação do indivíduo com outros e com o ambiente nos diferentes contextos gera diferentes situações de aprendizagens.

Diante da complexidade dos caminhos pelos quais perpassa a aprendizagem, o Modelo da Aprendizagem, com suas dimensões de aprendizagem, criado por Illeris (2007), facilita a compreensão e ajuda no processo de entendimento da aprendizagem experimentada pelas pessoas ao longo da vida. O modelo indica que todo o processo de aprendizagem e toda a sua natureza são determinados pelo contexto em que indivíduo se encontra inserido, sendo composto por três dimensões, nomeadamente o conteúdo, o incentivo e a interação (Figura 3) 
Figura 3: Dimensões da aprendizagem efetiva

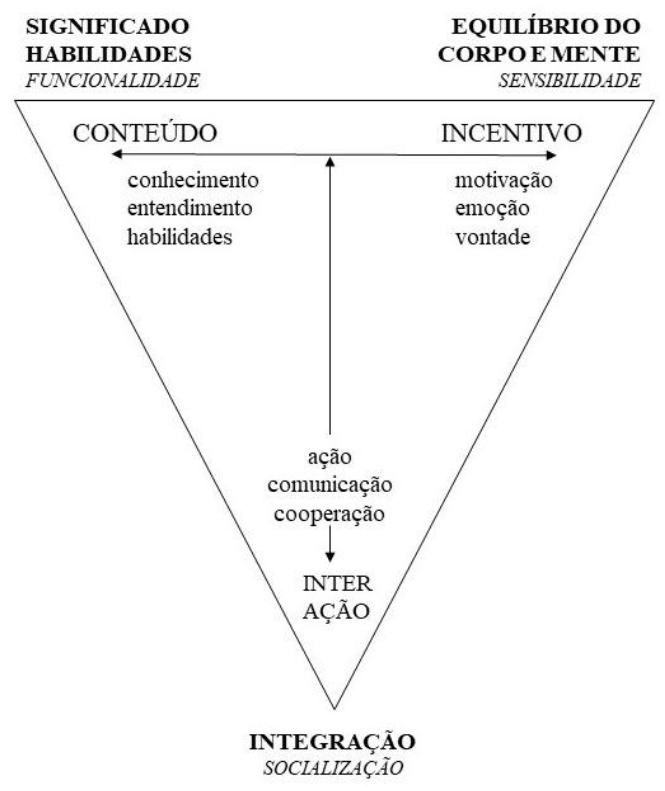

Fonte: Adaptado de Illeris (2007).

Em qualquer processo de aprendizagem, há conteúdos que apresentam, por exemplo, características relacionadas aos conhecimentos, habilidades, opiniões, entendimentos, instruções, significados, comportamentos, atitudes, qualificações e competências. Entretanto, os conteúdos também podem ser vistos por uma perspectiva ampla, envolvendo aquisição de cultura geral, métodos de trabalho ou, ainda, aspectos de "aprender a aprender".

A dimensão conteúdo da aprendizagem apresentada na Figura 3, de maneira geral, vai além da visão tradicional da aprendizagem presente no sistema educacional, em que o objetivo era meramente relacionado à aquisição de conhecimentos e habilidades. Primeiramente, é preciso entender que a aprendizagem pode tornar-se tudo aquilo que pode ser descrito com palavras e que, de fato, é inerente ao cérebro humano, a capacidade e a naturalidade de atribuir significado para aquilo que se aprende. Hoje o aprendizado envolve, dentre outras coisas, o aprender sobre si próprio, compreendendo as próprias reações, preferências, potencialidades e fraquezas (ILLERIS, 2007).

Já a dimensão incentivo da aprendizagem contempla aquilo que é normalmente relativo às emoções, às motivações e à vontade. Essa dimensão predispõe a base para mobilizar a energia que é necessária para a aprendizagem do indivíduo. Ela também se torna parte do processo de aprendizagem, influenciando na qualidade, como, por exemplo, na permanência e na utilidade. O processo de aquisição da aprendizagem raramente é um processo fácil, passando muitas vezes por momentos de distúrbios ou mesmo de desequilíbrio social, embora, na maioria dos casos, a motivação do indivíduo sempre esteja presente (ILLERIS, 2007). Obviamente, para se obter a aquisição da aprendizagem, é preciso existir afetividade na motivação e reconhecer que o gestor esportivo nunca irá resolver qualquer problema se este não o interessar.

Por fim, a dimensão interação terá inúmeras formas, como a percepção, a transmissão, a experiência, a imitação, a atividade ou a participação. Assim, é importante entender que, quanto maior o grau de interação na atividade, maiores serão as possibilidades de aprendizagem. No que diz respeito à forma mais direta e geral de interação, destacamos as comunidades 
de prática, que são traduzidas como o compartilhamento de aprendizagens em determinados grupos que se assemelham devido a suas rotinas, hábitos, crenças, entre outros (WENGER, 2008). Além disso, as comunidades de prática reproduzem a união do grupo da mesma maneira em que ele surgiu, compartilhando competências com as novas gerações pelo mesmo processo em que ela é desenvolvida.

$\mathrm{Na}$ tentativa de aproximar a aprendizagem ao contexto da gestão do esporte, há o reconhecimento da existência da aprendizagem gerencial, a qual considera o papel da aprendizagem como uma mudança de paradigma, ou seja, envolve o confronto de ideias e conceitos preestabelecidos a fim de que os resultados dessas novas aprendizagens sejam utilizados para a solução de problemas e tomadas de decisões (WENGER, 2008; CUNLIFFE; BELL, 2016). Além disso, ela rompe com a teoria tradicional da aprendizagem que considera como processos somente a transferência de conhecimentos (MORAES; SILVA; CUNHA, 2004).

O processo de aprendizagem no contexto gerencial indica que o diálogo, a experiência e a articulação entre as pessoas refletem diretamente na atuação. Também aponta que a articulação e a capacidade de transformação de conhecimentos comuns às novas ideias refletem no processo de construção do produto final ou de solução de problemas (LIU, 2015). Para que ocorra essa transformação da aprendizagem, é necessário que o indivíduo passe pelo processo de reflexão, podendo levar a processos muito mais profundos do que simples reflexões sobre as próprias práticas. No contexto da gestão, a reflexão precisa estimular questionamentos sobre o que é conhecimento e o que é apenas senso comum, o que é falado e o que não é, o que está sendo privilegiado ou marginalizado, múltiplas concepções e práticas, como também explorar formas de organizar, gerenciar e aprender (CUNLIFFE; BELL, 2016).

\section{Ambientes de aprendizagem profissional}

Toda a aprendizagem pode ser considerada inserida dentro de um contexto ou um ambiente (ILLERIS, 2007). De acordo com o modelo da sociedade moderna, em que as relações de trabalho influenciam a educação e a produção do conhecimento (DEETZ, 1992), em cada ambiente de aprendizagem existem particularidades, das quais são destacados neste ensaio os ambientes educacional e do trabalho. Entretanto, antes de adentrar nos ambientes de aprendizagem, há de se destacar que o contexto esportivo se apresenta especial no que diz respeito à trajetória de grande parte dos gestores esportivos (CRUZ, 2017). As atuações como atleta, treinador e árbitro são partes integrantes desse contexto. No caso da atuação como atleta, destaca-se a competência de autodeterminação. Essa competência demonstra ser um fator muito importante e predominante durante a atuação como atleta, podendo ser considerada fundamental para a eficiência e a eficácia dos resultados como atleta (LONSDALE; HODGE; ROSE, 2009; DEFREESE; SMITH, 2013; MARTINENT; DECRET, 2015; MONTEROCARRETERO et al., 2015), o que pode ter continuidade em uma atuação no pós-carreira quando o ex-atleta é promovido (capacitado de fato ou não) para o papel de dirigente esportivo (JOAQUIM; BATISTA; CARVALHO, 2011; CRUZ, 2017).

$\mathrm{Na}$ atuação como treinador, são definidas inúmeras competências centrais que são atribuídas também com a atuação futura como gestor do esporte, como definir visão estratégica, planejar, buscar objetivos, construir relações, entre outros (ICCE, 2013; MILISTETD et al., 2017). Além disso, a competência de compreender o ambiente de prática é considerada um dos elementos do conhecimento profissional que o treinador e demais profissionais de Educação Física e Esporte devem dominar (ICCE, 2012), o que pode indicar que esse conhecimento foi aprendido e transformado em competência na atuação como gestor. No caso da atuação como árbitro, é verificada a comunicação como sendo uma das competências essenciais para esse tipo de função (ANSHEL, 1995; SIMMONS, 2010; 
SOUCHON et al., 2016), além de precisar demonstrar competência pedagógica ao ter que saber explicar claramente suas decisões (ISIDORI; MÜLLER; KAYA, 2012).

Sobre o contexto do esporte no Brasil, após a década dos megaeventos, a percepção predominante é de "terra arrasada", especialmente devido às falsas crenças em relação aos supostos benefícios e sua falta de planejamento para seu desenvolvimento futuro (COAKLEY; SOUZA, 2015). Pontos positivos foram alcançados, relacionados com legislações sobre o financiamento do esporte, a melhoria nas estruturas esportivas de algumas cidades e outros poucos que podem ser identificados. Mas, a partir do ponto de vista da gestão do esporte, não se conseguiu um impacto/legado no sistema esportivo brasileiro. Constatamos de forma prática e concreta a ausência de uma gestão profissional, ética e responsável, assim como a continuação da perspectiva de descontinuidade nos programas implementados, a falta de visão estratégica, a ineficácia, a irresponsabilidade e a ineficiência na utilização dos recursos disponíveis. Também é possível constatar que, no atual modelo brasileiro, os profissionais envolvidos na direção das principais organizações esportivas (principalmente confederações) não têm credibilidade (MAZZEI; ROCCO JÚNIOR, 2017). E resta a pergunta: por onde se deve partir para assegurar a melhoria deste cenário?

\section{Ambiente de aprendizagem educacional}

É o ambiente educacional que possibilitará as oportunidades de uma aprendizagem aprofundada, principalmente quando se tratar de assuntos complexos e de percepções teóricas. Esse ambiente é caracterizado por apresentar processos de aprendizagem formal, racional e direcionada. A aprendizagem educacional geralmente e atualmente é uma das mais relevantes e aceitas pela sociedade, pois é nela que o indivíduo terá o seu direcionamento para o campo de trabalho (ILLERIS, 2011). Em termos de aprendizagem nesse ambiente, é crucial que exista a relação professor/tutor e estudante, na qual o conhecimento será de alguma forma transferido ou descoberto (ILLERIS, 2007). São consideradas situações de aprendizagem desse ambiente aquelas em que o indivíduo se encontra inserido em escolas, universidades, cursos de longa duração e atividades educacionais, sistematizadas e organizadas.

Um dos desafios iniciais de toda e qualquer aprendizagem no ambiente educacional é que ela se conecte com o mundo real. Ou seja, que nesse ambiente de aprendizagem seja levada em conta a relação teoria e prática (ILLERIS, 2009). A necessidade de se investigar os cursos de formação de gestores esportivos tem se propagado nos últimos dez anos (BASTOS, 2004), sendo que a busca pela adequação curricular deveria ir ao encontro das complexas e variadas necessidades do esporte contemporâneo (DESENSI et al., 1990).

Considerando o ambiente educacional do gestor esportivo brasileiro, a sua formação inicial está geralmente atrelada à área de Educação Física e Esporte (GOMES; SARMENTO; PEDROSO, 2014; AMARAL; BASTOS, 2015) e a gestão esportiva é apontada como um dos campos de atuação desses profissionais (CONFEF, 2002). Por outro lado, parece se fazer necessário que as estruturas curriculares da área de Educação Física e Esporte considerem aprendizagens para tal atuação profissional do gestor esportivo, o que não parece ser a realidade em boa parte dos cursos (FEITOSA; NASCIMENTO, 2006; QUINAUD; FARIAS; NASCIMENTO, no prelo). Além disso, embora existam resoluções (BRASIL, 2004; 2009) que regulamentem os cursos de graduação em Educação Física e Esporte no Brasil, a formação do gestor esportivo, bem como de suas competências a serem adquiridas no decorrer do curso não são priorizadas em virtude de uma perspectiva generalista de formação. Na prática, uma boa porcentagem dos gestores esportivos no Brasil tem diferentes formações acadêmicas, se perpetuando no país a cultura do cartola (MAZZEI; ROCCO JÚNIOR, 2017).

Um aspecto a ressaltar é a necessidade de mais estudos direcionados a investigar o contexto da gestão esportiva nas disciplinas ofertadas nos cursos de formação em Educação 
Física e Esporte no Brasil (QUINAUD; FARIAS; NASCIMENTO, no prelo). Vale ressaltar que o campo de atuação da gestão do esporte não é somente dos profissionais de Educação Física e Esporte, nem tampouco de uma determinada profissão e que, independentemente da formação, o que deve ser considerado são as aprendizagens inerentes à atuação de um gestor do esporte. Apesar disso, uma das preocupações de o esporte ser gerenciado por profissionais sem a formação em Educação Física e Esporte é a utilização do esporte apenas como ferramenta mercadológica (PIRES; LOPES, 2001).

Não obstante, a falta de aprendizagens necessárias para sua atuação já é um fator evidenciado nos currículos da formação inicial em Educação Física e Esporte, em que os conteúdos da gestão são compactados em uma única disciplina ou no máximo duas (CÁRDENAS; FEUERSCHÜTTE, 2015). Além da falta de disciplinas de gestão nos currículos de formação, há outro agravante de sua formação, uma vez que ainda não existem docentes qualificados e em número suficiente para o próprio ensino da gestão esportiva na área de Educação Física e Esporte no Brasil (MAZZEI; ROCCO JÚNIOR, 2017). Apesar da incipiência de disciplinas e de lacunas referentes às aprendizagens da gestão nos currículos de formação, podemos dizer que mesmo assim a gestão tem conquistado seu espaço paulatinamente na Educação Física e Esporte no país, principalmente em instituições de vanguarda, que oferecem tanto cursos de graduação como programas de pós-graduação, e que alcançam ótima avaliação nos órgãos nacionais responsáveis por essas duas áreas no país (MONTAGNER; SCAGLIA; AMAYA, 2012; MAZZEI; AMAYA; BASTOS, 2013).

Além dos cursos de formação inicial, os cursos de pós-graduação (lato sensu) e atualização também são considerados parte do ambiente educacional. No contexto da pósgraduação, as aprendizagens são consideradas mais específicas do que um curso de graduação, o que proporciona ao indivíduo um possível processo de autorreflexão em relação ao contexto social/profissional em que se encontra (CONKLIN; KYLE; ROBERTSON, 2012). A pós-graduação auxilia na aquisição de competências que podem ser úteis aos gestores (RUAS; COMINI, 2007). Mesmo que o curso não tenha foco na gestão esportiva, cursos de pósgraduação em administração podem proporcionar a aquisição de competências como comunicação, autoconfiança e planejamento, além de networking com outros profissionais (HINCHLIFFE; JOLLY, 2011; JACKSON, 2014). No Brasil, as opções de cursos específicos em gestão do esporte têm se proliferado, mas não há um levantamento detalhado sobre o número, a existência de fato e a qualidade desses cursos.

No que diz respeito à pós-graduação stricto sensu, não há cursos relacionados com a temática da gestão esportiva no Brasil. O que existe são linhas de pesquisa em programas de Educação Física e Esporte com foco na gestão do esporte ou com temas correlacionados com essa área. Em vista deste cenário do ambiente de aprendizagem educacional e a formação do gestor esportivo no Brasil, os conteúdos de aprendizagem específicos para atuação profissional como gestor esportivo são provavelmente adquiridos ao longo do percurso profissional (BRIDGSTOCK, 2009).

\section{Ambiente de aprendizagem no trabalho}

O ambiente de aprendizagem no trabalho pode ser considerado especial e significante por proporcionar oportunidades específicas e condições de aprendizagem para a atuação profissional, mesmo que o processo de aprendizagem não seja o principal foco desse ambiente (ILLERIS, 2011). As situações que envolvem os membros da organização, seja por conversas informais, observação, tomada de decisão, seja por solução de problemas, podem ser consideradas fundamentais para o crescimento do profissional (HOOVER; GIAMBATISTA; BELKIN, 2012; LETMATHE, SCHWEITZER; ZIELINSKI, 2012). 
Dentre os processos de aprendizagem em um ambiente de trabalho, podem-se destacar os relacionamentos interpessoais entre os membros da organização, pois a partir dessas interações é possível entender a dinâmica dos grupos e dimensionar com maior profundidade as consequências das tomadas de decisões (BENSON; LORENZONI; COOK, 2016). Além disso, a adequada convivência com os pares promove a oportunidade de se desenvolver novas aprendizagens (VAN VEELEN; SLEEGERS; ENDEDIJK, 2017), bem como incentiva o sujeito a refletir acerca de suas ações em prol de um pensamento que contribua com os anseios de todos os outros profissionais com quem convive (FENWICK, 2008).

Outra situação de aprendizagem observada comumente em ambiente de trabalho compreende os "erros", quando ocorre uma aprendizagem a partir do reconhecimento e da reflexão sobre as falhas. Assim, o bom convívio entre os membros é fundamental para a aquisição de novas aprendizagens, porque, caso ocorram críticas severas em relação ao erro, o sujeito pode acabar sendo desestimulado em realizar novas ações. De acordo com Cope (2011), aprender com os erros é fundamental para um profissional mais eclético em uma gestão do esporte que, por natureza, impõe diferentes desafios para seus gestores.

Apesar de a aprendizagem pelo erro ser um processo importante para o gestor do esporte (QUINAUD; MILISTETD; NASCIMENTO, no prelo), ele pode acabar prejudicando a organização da qual faz parte, uma vez que as ações ali presentes podem não ser efetivas. Em vista disso, é de se considerar que sejam incentivadas ações do ambiente de trabalho pautadas em uma base consolidada de estudos e pesquisas, pois os problemas majoritariamente encontrados no ambiente de trabalho são provenientes da falta de aprendizagem no ambiente educacional ou do modo em que essas aprendizagens são transmitidas, além da questão de caráter e do comportamento pessoal (FRISBY, 2005).

Infelizmente, muito do ambiente de aprendizagem no trabalho do gestor esportivo no Brasil é pautado no senso comum ou, ainda, em um conjunto de experiências que já não condizem com o atual contexto do esporte. E, ainda, é necessário citar que, em muitos casos, a perpetuação no poder ou o desejo de permanência em cargos consideráveis fazem com que bons gestores caiam na vala comum do estigma de "cartola". Os casos comprovados de irregularidades envolvendo as principais organizações esportivas brasileiras apenas confirmam tais afirmações. Existem exceções, sim, mas elas ainda são raras ou acabam por ser isoladas e ainda descontinuadas em um sistema cruel.

Além disso, os futuros profissionais deveriam considerar melhor o entendimento dos problemas da prática profissional, para assim compreenderem as suas dificuldades e limitações e então desenvolverem aprendizagens com foco crítico para a solução desses problemas (INGLIS, 2007). Acreditamos que apenas com a união do conhecimento proveniente de diferentes ambientes de aprendizagem existirá a formulação de ideias e soluções de problemas mais abrangentes, e a gestão do esporte se desenvolveria com o propósito de alcançar melhor qualidade em suas ações (MAHONY, 2008).

A fim de melhor esclarecer o pensamento em torno da necessidade de unir as aprendizagens do ambiente educacional com o ambiente de trabalho, pode ser citado o exemplo dos Estados Unidos, que contam com aproximadamente 415 cursos de graduação, 172 programas de mestrado e 27 programas de doutorado específicos em gestão do esporte (MAZZEI; ROCCO JÚNIOR, 2017) e que defendem que a aprendizagem só tem sentido se for praticada ao mesmo tempo na academia e fora dela. Obviamente que há muito mais diferenças do que somente aquelas identificadas no sistema esportivo, mas não se pode negar que o sistema norte-americano é um dos mais efetivos em termos de gestão do esporte. 


\section{Considerações finais}

Entendemos que o gestor esportivo se apresenta como um indivíduo responsável por inúmeras atribuições e que, para tal, deve ser dotado de competências e habilidades, muitas vezes exigidas pelas próprias funções do cargo. Por mais que se tente descrever um perfil mais atual do gestor esportivo, as características refletem as particularidades de cada um, assim como geralmente fazem sentido no respectivo contexto, o que faz a análise de um perfil ser sempre particular com o tipo de organização e sua gestão analisada. Não defendemos aqui uma gestão do esporte mercadológica, como erroneamente muitos acadêmicos e outros profissionais interpretam. Mas sim um esporte melhor organizado e que atenda às necessidades da população. Assim, acredita-se que os profissionais formados em Educação Física e Esporte, que estudam os valores positivos presentes no esporte e seu potencial de desenvolvimento humano, sejam os mais indicados para ocuparem cargos diretivos de gestores do esporte.

Ao mesmo tempo surge a reflexão acerca da necessidade da inclusão de mais conteúdos de gestão nos cursos de graduação em Educação Física e Esporte para que sejam abordados transversalmente nos currículos de formação, o que não significa apenas a criação de mais disciplinas para as grades curriculares, mas também o desenvolvimento de uma quantidade e de uma qualidade consistentes de cursos de pós-graduação coerentes com a atuação profissional na área do esporte em geral. Ressalta-se que não estamos querendo dizer que é apenas o profissional de Educação Física e Esporte que tem a responsabilidade de atuar nessa área e nem que os cursos de formação inicial em Educação Física e Esporte devem ter como foco principal a gestão do esporte, porém, que é essencial uma reflexão sobre a formação dos gestores esportivos em virtude de uma nova realidade social e o papel do esporte neste contexto.

Apesar da importância da aprendizagem em ambiente de trabalho, as chances de que a atuação se torne mais efetiva vão ao encontro das aprendizagens acumuladas pelo indivíduo ao longo do tempo e, principalmente, com a sua formação educacional. Infelizmente, a gestão do esporte no ambiente de aprendizagem educacional foi desprezada durante anos no Brasil, assim como o ambiente de aprendizagem do trabalho foi dominado por muito mais erros do que um processo de melhoria continuada.

Por fim, acreditamos que este ensaio irá auxiliar na reflexão acerca do gestor esportivo e contribuir para que novos estudos apresentem abordagens mais diversificadas e profundas. A área de investigação sobre aprendizagem de gestores, internacionalmente, já está em grande desenvolvimento, mas ainda há uma lacuna em relação aos gestores esportivos e, principalmente, na literatura brasileira.

\section{Referências}

AMARAL, C. M. D. S.; BASTOS, F. D. C. O gestor esportivo no Brasil: revisão de publicações no país. Revista Intercontinental de Gestão Desportiva, v. 5, n. 1, p. 68-78, jun. 2015.

ANSHEL, M. H. Development of a rating scale for determining competence in basketball referees: Implications for sport psychology. The Sport Psychologist, v. 9, n. 1, p. 4-28, mar. 1995.

AZEVÊDO, P. H.; BARROS, J. D. F.; SUAIDEM, S. Caracterização do perfil do gestor esportivo dos clubes da primeira divisão de futebol do Distrito Federal e suas relações com a legislação esportiva brasileira. Journal of Physical Education, v. 15, n. 1, p. 33-42, 2004. 
BARROS FILHO, M. A. et al. Perfil do gestor esportivo brasileiro: uma revisão de literatura. Revista Intercontinental de Gestão Desportiva, v. 3, dez. 2013.

BASTOS, F. C. Administração esportiva: área de estudo, pesquisa e perspectivas no Brasil. Motrivivência, v. 15, n. 20-21, p. 295-306, 2004.

BASTOS, F. C. et al. Perfil do administrador esportivo de clubes de São Paulo/Brasil. Revista Mackenzie de Educação Física e Esporte, v. 5, n. 1, p. 13-22, 2006.

BENSON, D.; LORENZONI, I.; COOK, H. Evaluating social learning in England flood risk management: an 'individual-community interaction'perspective. Environmental Science \& Policy, v. 55, n. 1, p. 326-334, jan. 2016.

BENTO, J. O.; CONSTANTINO, J. M. Em defesa do desporto: mutações e valores em conflito. Coimbra: Almedina, 2007.

BRASIL. Resolução $\mathrm{n}^{\circ}$ 7, de 31 de março de 2004. Institui as Diretrizes Curriculares Nacionais para os cursos de graduação em Educação Física, em nível superior de graduação plena. Diário Oficial da União. Brasília, 2004.

Resolução n. 4, de 6 de abril de 2009. Dispõe sobre carga horária mínima e procedimentos relativos à integralização e duração dos cursos de graduação em Biomedicina, Ciências Biológicas, Educação Física, Enfermagem, Farmácia, Fisioterapia, Fonoaudiologia, Nutrição e Terapia Ocupacional, bacharelados, na modalidade presencial. Diário da União, Brasília, 7 de abril de 2009. Disponível em: <http://portal.mec.gov.br/dmdocuments/rces004_09.pdf.2009>. Acesso em: 3 mar. 2018.

BRIDGSTOCK, R. The graduate attributes we've overlooked: Enhancing graduate employability through career management skills. Higher Education Research \& Development, v. 28, n. 1, p. 31-44, feb. 2009.

CÁRDENAS, A. R.; FEUERSCHÜTTE, S. G. A gestão na formação inicial em Educação Física: um olhar qualitativo sobre currículos, disciplinas e ementas dos cursos de bacharelado de Santa Catarina. Criar Educação, v. 4, n. 1, p. 10-26, jan./jun. 2015.

CAUPIN, G. et al. ICB - IPMA Competence Baseline. Netherlands: International Project Management Association, 2006.

COAKLEY, J.; SOUZA, D. L. Legados de megaeventos esportivos: considerações a partir de uma perspectiva crítica. Revista Brasileira de Educação Física e Esportes, v. 29, n. 4, p. 675-86, 2015.

CONFEF. Resolução n. 46, de 18 de fevereiro de 2002. Dispõe sobre a intervenção do profissional de Educação Física e respectivas competências e define os seus campos de atuação profissional. Rio de Janeiro, 18 de fevereiro de 2002. Disponível em: <http://www.confef.org.br/extra/resolucoes/conteudo.asp?cd_resol=82>. Acesso em: 11 abr. 2017. 
CONKLIN, J.; KYLE, T.; ROBERTSON, C. The essential transformation: How Masters students make sense and learn through transformative change. Management Learning, v. 44, n. 2, p. 161-178, mar. 2012.

COPE, J. Entrepreneurial learning from failure: An interpretative phenomenological analysis. Journal of Business Venturing, v. 26, n. 6, p. 604-623, 2011.

CRUZ, I. H. Histórias de sucesso na gestão do desporto: O percurso e o perfil de quatro gestores. 2017. 152 f. Dissertação (Mestrado) - Universidade do Porto, Porto, Portugal, 2017.

CUNLIFFE, A.; BELL, E. Management learning: legacies and future possibilities. London, UK: SAGE Publications Sage, 2016.

DEETZ, S. Democracy in an age of corporate colonization: developments in communication and the politics of everyday life. New York: SUNY Press, 1992.

DEFREESE, J.; SMITH, A. L. Teammate social support, burnout, and self-determined motivation in collegiate athletes. Psychology of Sport and Exercise, v. 14, n. 2, p. 258-265, mar. 2013.

DESENSI, J. T. et al. Sport management curricular evaluation and needs assessment: A multifaceted approach. Journal of Sport Management, v. 4, n. 1, p. 31-58, jan. 1990.

FEITOSA, W. M. N.; NASCIMENTO, J. V. D. Educação Física: quais competências profissionais? In: NETO, S. S.; HUNGER, D. (Ed.). Formação profissional em Educação Física: estudos e pesquisas. Rio Claro: Bioética, 2006. p. 87-108.

FELIX, C. A. F.; FERNANDES, A. S.; OLIVEIRA, P. W. S. Competências gerenciais da equipe do comitê organizador dos jogos Pan-Americanos Rio 2007. Catussaba - Revista Científica da Escola da Saúde, v. 4, n. 3, p. 19-26, 2015.

FENWICK, T. Understanding relations of individual - collective learning in work: a review of research. Management Learning, v. 39, n. 3, p. 227-243, jul. 2008.

FREITAS, D.; GIRGINOV, V.; TEOLDO, I. What do they do? Competency and managing in Brazilian Olympic Sport Federations. European Sport Management Quarterly, v. 17, n. 2, p. 193-209, sep. 2016.

FREITAS, D. M. et al. Liderança dos presidentes das federações olímpicas brasileiras: Análise da autopercepção das competências em função da formação académica. Revista Portuguesa de Ciências do Desporto, v. 15, n. 1, p. 79-98, 2015.

FRISBY, W. The good, the bad, and the ugly: critical sport management research. Journal of Sport Management, v. 19, n. 1, p. 1-12, jan. 2005.

GOMES, J. L. D. B.; SARMENTO, J. P.; PEDROSO, C. A. M. Q. Competências, perfil e formação do gestor esportivo de academia de ginástica: uma revisão. Resvista Intercontinental de Gestão Desportiva, v. 4, n. 1, p. 99-112, jun. 2014. 
HINCHLIFFE, G. W.; JOLLY, A. Graduate identity and employability. British Educational Research Journal, v. 37, n. 4, p. 563-584, jan. 2011.

HOOVER, J. D.; GIAMBATISTA, R. C.; BELKIN, L. Y. Eyes on, hands on: Vicarious observational learning as an enhancement of direct experience. Academy of Management Learning \& Education, v. 11, n. 4, p. 591-608, jul. 2012.

ILLERIS, K. How we learning: learning and non-learning in school and beyond. New York: Routledge, 2007.

Transfer of learning in the learning society: How can the barriers between different learning spaces be surmounted, and how can the gap between learning inside and outside schools be bridged? International Journal of Lifelong Education, v. 28, n. 2, p. 137-148, mar. 2009.

. The fundamental of workplace learning: undertanding how people learn in working place. London: Routledge, 2011.

INGLIS, S. Creative tensions and conversations in the academy. Journal of Sport Management, v. 21, n. 1, p. 1-14, jan. 2007.

INTERNATIONAL COUNCIL FOR COACHING EXCELLENCE (ICCE). International Sport Coaching Framework version 1.1. Champaign: Human Kinetics, 2012.

\section{Kinetics, 2013.}

International Sport Coaching Framework Version 1.2. Champaign: Human

ISIDORI, E.; MÜLLER, A.; KAYA, S. The referee as educator: hermeneutical and pedagogical perspectives. Physical Culture and Sport. Studies and Research, v. 56, n. 1, p. $5-11,2012$.

JACKSON, D. Skill mastery and the formation of graduate identity in bachelor graduates: evidence from Australia. Studies in Higher Education, v. 41, n. 7, p. 1313-1332, nov. 2014.

JARVIS, P. Towards a comprehensive theory of human learning. Abingdon: Routledge, 2006.

Globalisation, lifelong learning and the learning society: sociological perspectives. New York: Routledge, 2007.

Democract, lifelong learning and the learning society: active citizenship in a late modern age. New York: Routledge, 2008.

JOAQUIM, B. A.; BATISTA, P. M.; CARVALHO, M. J. Revisão Sistemática sobre o perfil de competências do gestor desportivo. Movimento, v. 17, n. 1, p. 255, jan./mar. 2011.

JÚNIOR, A. R.; PADEIRO, C. Opinião: onde estão os verdadeiros gestores de esporte no nosso futebol? Disponível em: <https://esporte.uol.com.br/ultimas-noticias/esporte-pontofinal/2018/01/05/opiniao-onde-estao-os-verdadeiros-gestores-do-esporte-

brasileiro.htm?cmpid=copiaecola, 2018>. Acesso em: 8 jan. 2018. 
KARNAS, G. S. Perfil do gestor esportivo nos países de língua portuguesa: uma revisão de literatura. 2010. 36 f. Monografia (Graduação) - Universidade Federal do Rio Grande do Sul, Porto Alegre, 2010.

LETMATHE, P.; SCHWEITZER, M.; ZIELINSKI, M. How to learn new tasks: Shop floor performance effects of knowledge transfer and performance feedback. Journal of Operations Management, v. 30, n. 3, p. 221-236, mac. 2012.

LIU, R. Management learning in business networks: The process and the effects. Management Learning, v. 46, n. 3, p. 337-360, jun. 2015.

LONSDALE, C.; HODGE, K.; ROSE, E. Athlete burnout in elite sport: A self-determination perspective. Journal of Sports Sciences, v. 27, n. 8, p. 785-795, jun. 2009.

MAHONY, D. F. No one can whistle a symphony: Working together for sport management's future. Journal of Sport Management, v. 22, n. 1, p. 1-10, jan. 2008.

MARJORIBANKS, T.; FARQUHARSON, K. Contesting competence chief executive officers and leadership in Australian Football League clubs. Marketing Intelligence \& Planning, v. 34, n. 2, p. 188-202, 2016.

MARTINENT, G.; DECRET, J.-C. Motivational profiles among young table-tennis players in intensive training settings: A latent profile transition analysis. Journal of Applied Sport Psychology, v. 27, n. 3, p. 268-287, mar. 2015.

MAZZEI, L. C.; AMAYA, K.; BASTOS, F. C. Programas acadêmicos de graduação em gestão do esporte no Brasil. Revista Mackenzie de Educação Física e Esporte, v. 12, n. 1, p. 219-234, 2013.

MAZZEI, L. C. et al. Centros de treinamento esportivo para o esporte de alto rendimento no Brasil: um estudo preliminar. Revista Mineira de Educação Física, v. S1, p. 1575-1584, jan. 2012.

MAZZEI, L. C.; ROCCO JÚNIOR, A. J. Um ensaio sobre a gestão do esporte: um momento para a sua afirmação no Brasil. Revista de Gestão e Negócios do Esporte, v. 2, n. 1, p. 96109, maio 2017.

MELlO, J. A. C.; SILVA, S. A. P. S. Competências do gestor de academias esportivas. Motriz, v. 19, n. 1, p. 74-83, mar. 2013.

MILISTETD, M. et al. Formação de treinadores esportivos: orientações para a organização das práticas pedagógicas nos cursos de bacharelado em Educação Física. Revista da Educação física/UEM, v. 28, n. 2, 2017.

MILNE, R. M. Case studies on the changing roles, skills, and competence of school sport management. 2007. (PhD). Griffith University, Queensland.

MIRANDA, Y. D. H. B. et al. Análise acerca das competências necessárias para a atuação profissional do gestor esportivo. Pensar a Prática, v. 20, n. 3, p. 593-603, 2017. 
MOCSÁNYI, V.; BASTOS, F. C. Gestão de pessoas na administração esportiva: considerações sobre os principais processos. Revista Mackenzie de Educação Física e Esporte, v. 4, n. 4, p. 55-69, 2005.

MONTAGNER, P. C.; SCAGLIA, A. J.; AMAYA, K. G. Desafios da formação em esporte para intervenção profissional no contexto da gestão: investigações iniciais. In: NASCIMENTO, J. V.; FARIAS, G. O. (Ed.). Construção da identidade profissional em Educação Física: da formação à intervenção. Florianópolis: UDESC, 2012.

MONTERO-CARRETERO, C. et al. Motivación, estado de ánimo y flow en judocas de élite. Revista Mexicana de Psicología, v. 32, n. 2, p. 101-112, jul./dez. 2015.

MORAES, L. V. D. S. D.; SILVA, M. A. D.; CUNHA, C. J. C. A. Aprendizagem gerencial: teoria e prática. RAE-eletrônica, v. 3, n. 1, p. 1-21, jan./jun. 2004.

PACESA, N.; ZAKAREVICIUS, P.; ZUKAUSKAS, P. The profile of Lithuanian manager. Problems and perspectives in management, v. 9, n. 1, p. 59-70, 2011.

PIRES, G. M. V. D. S. Gestão do desporto: desenvolvimento organizacional. Porto: APOGESD, 2005.

PIRES, G. M. V. D. S.; LOPES, J. P. S. D. R. Conceito de gestão do desporto. Novos desafios, diferentes soluções. Revista Portuguesa de Ciências do Desporto, v. 1, n. 1, p. 88$103,2001$.

QUINAUD, R. T.; FARIAS, G. O.; NASCIMENTO, J. V. D. Formação profissional do gestor esportivo para o mercado de trabalho: a (in)formação dos cursos de bacharelado em educação física do Brasil. Movimento, no prelo.

QUINAUD, R. T.; MILISTETD, M.; NASCIMENTO, J. V. Situações de aprendizagem profissional no ambiente de trabalho de gestores de federações esportivas catarinenses. Journal of Physical Education, no prelo.

RUAS, R.; COMINI, G. M. Aprendizagem e desenvolvimento de competências: articulando teoria e prática em programas de pós-graduação em formação gerencial. Cadernos EBAPE. br, v. 5, n. SPE, p. 1-14, jan. 2007.

SCHWAB, K. A. et al. Career paths in sport management. Schole, v. 30, n. 2, p. 1-11, 2015.

SILVA, S. L. D. Gestão do conhecimento: uma revisão crítica orientada pela abordagem da criação do conhecimento. Ciências e Informação, v. 33, n. 2, p. 143-151, maio/ ago. 2004.

SIMMONS, P. Communicative displays as fairness heuristics: strategic football referee communication. Australian Journal of Communication, v. 37, n. 1, p. 75-94, mar. 2010.

SKINNER, J.; GILBERT, K. Sport management education: teaching and learning for the future. Sport Management Review, v. 10, n. 2, p. 125-131, sep. 2007. 
SMART, B. Not playing around: global capitalism, modern sport and consumer culture. Global Networks, v. 7, n. 2, p. 113-134, mai. 2007.

SORDI, J. D.; THEOBALD, R. R. As habilidades dos gestores esportivos: um estudo de caso em novo Hamburgo. Gestão e Desenvolvimento, v. 14, n. 2, p. 141-154, jun./dez. 2017.

SOUCHON, N. et al. Influence of competition level on referees' decision-making in handball. Social Influence, v. 11, n. 4, p. 246-258, nov. 2016.

TAVARES, A. L. As competências dos gestores públicos de esporte. 2010. Dissertação (Mestrado) - Fundação Cultural Dr. Pedro Leopoldo, Pedro Leopoldo, 2010.

VAN VEELEN, R.; SLEEGERS, P. J. C.; ENDEDIJK, M. D. Professional learning among school leaders in secondary education: the impact of personal and work context factors. Educational Administration Quarterly, v. 53, n. 3, p. 365-408, jan. 2017.

VILHELMSDOTTIR, A. S.; KRISTJANSDOTTIR, E.; INGASON, H. T. What can be learnt from the Icelandic National Team's Managers, in Project Management and in Shaping Group Dynamics? Procedia-Social and Behavioral Sciences, v. 226, p. 278-285, jul. 2016.

WENGER, E. Communities of practice: learning, meaning, and identity. NewYork: Cambridge University Press, 2008.

Recebido em: 29/03/2018

Revisado em: 21/05/2018

Aprovado em: 03/08/2018

Endereço para correspondência:

ricardoquinaud@gmail.com

Ricardo Teixeira Quinaud

Universidade Federal de Santa Catarina-Câmpus Trindade

Centro de Desportos

Av. César Seara - Carvoeira

Florianópolis - SC, Brasil 